\title{
Power sums of Hecke eigenvalues and application
}

by

\section{J. Wu (Jinan and Nancy)}

1. Introduction. Let $k \geq 2$ be an even integer and $N \geq 1$ be squarefree. Denote by $\mathrm{H}_{k}^{*}(N)$ the set of all normalized Hecke primitive eigencuspforms of weight $k$ for the congruence modular group

$$
\Gamma_{0}(N):=\left\{\left(\begin{array}{ll}
a & b \\
c & d
\end{array}\right) \in \mathrm{SL}_{2}(\mathbb{Z}): c \equiv 0(\bmod N)\right\} .
$$

Here the normalization is taken to have $\lambda_{f}(1)=1$ in the Fourier series of $f \in \mathrm{H}_{k}^{*}(N)$ at the cusp $\infty$,

$$
f(z)=\sum_{n=1}^{\infty} \lambda_{f}(n) n^{(k-1) / 2} e^{2 \pi i n z} \quad(\operatorname{Im} z>0) .
$$

Inherited from the Hecke operators, the normalized Fourier coefficient $\lambda_{f}(n)$ satisfies the relation

$$
\lambda_{f}(m) \lambda_{f}(n)=\sum_{\substack{d \mid(m, n) \\(d, N)=1}} \lambda_{f}\left(\frac{m n}{d^{2}}\right)
$$

for all integers $m, n \geq 1$. In particular, $\lambda_{f}(n)$ is multiplicative.

Following Deligne [4], for any prime number $p$ there are two complex numbers $\alpha_{f}(p)$ and $\beta_{f}(p)$ such that

$$
\begin{cases}\alpha_{f}(p)=\varepsilon_{f}(p) p^{-1 / 2}, \quad \beta_{f}(p)=0 & \text { if } p \mid N, \\ \left|\alpha_{f}(p)\right|=\alpha_{f}(p) \beta_{f}(p)=1 & \text { if } p \nmid N,\end{cases}
$$

and

$$
\lambda_{f}\left(p^{\nu}\right)=\frac{\alpha_{f}(p)^{\nu+1}-\beta_{f}(p)^{\nu+1}}{\alpha_{f}(p)-\beta_{f}(p)}
$$

2000 Mathematics Subject Classification: 11F30, 11F66.

Key words and phrases: Fourier coefficients of automorphic forms, Dirichlet series. 
for all integers $\nu \geq 1$, where $\varepsilon_{f}(p)= \pm 1$. Hence $\lambda_{f}(n)$ is real and satisfies Deligne's inequality

$$
\left|\lambda_{f}(n)\right| \leq d(n)
$$

for all integers $n \geq 1$, where $d(n)$ is the divisor function. In particular, for each prime number $p \nmid N$ there is $\theta_{f}(p) \in[0, \pi]$ such that

$$
\lambda_{f}(p)=2 \cos \theta_{f}(p) .
$$

See e.g. [9] for basic analytic facts about modular forms.

Positive real moments of Hecke eigenvalues were first studied by Rankin ([16], [17]). For $f \in \mathrm{H}_{k}^{*}(N)$ and $r \geq 0$, consider the sum of the $2 r$ th powers of $\left|\lambda_{f}(n)\right|$ :

$$
S_{f}^{*}(x ; r):=\sum_{n \leq x}\left|\lambda_{f}(n)\right|^{2 r} .
$$

The method of Rankin [17] illustrates how to obtain optimal lower and upper bounds for $S_{f}^{*}(x ; r)$ if we only know that the associated Dirichlet series

$$
F_{r}(s):=\sum_{n \geq 1}\left|\lambda_{f}(n)\right|^{2 r} n^{-s} \quad(\operatorname{Re} s>1)
$$

is invertible for $\operatorname{Re} s \geq 1$ (i.e. holomorphic and nonzero for $\operatorname{Re} s \geq 1$ ) when $r=1,2$. (The invertibility in these two cases is known by Moreno \& Shahidi [15].) Rankin's result ([17, Theorem 1]) states that

$$
x(\log x)^{\delta_{r}^{\mp}} \ll S_{f}^{*}(x ; r) \ll x(\log x)^{\delta_{r}^{ \pm}} \quad\left(r \in \mathcal{R}^{\mp}\right)
$$

for $x \geq x_{0}(f, r)$, where

$$
\mathcal{R}^{-}:=[0,1] \cup[2, \infty), \quad \mathcal{R}^{+}:=[1,2],
$$

and

$$
\delta_{r}^{-}:=2^{r-1}-1, \quad \delta_{r}^{+}:=\frac{2^{r-1}}{5}\left(2^{r}+3^{2-r}\right)-1 .
$$

The implied constants in (1.9) depend on $f$ and $r$.

On the other hand, if the Sato-Tate conjecture holds for a newform $f$, then

$$
S_{f}^{*}(x ; r) \sim C_{r}(f) x(\log x)^{\theta_{r}} \quad(x \rightarrow \infty),
$$

where $C_{r}(f)$ is a positive constant depending on $f, r$, and

$$
\theta_{r}:=\frac{4^{r} \Gamma(r+1 / 2)}{\sqrt{\pi} \Gamma(r+2)}-1 .
$$

We remark that this conjecture has been proved for elliptic curves over $\mathbb{Q}$ with multiplicative reduction at some prime (cf. $[1,21,7])$. 
Very recently, Tenenbaum [23] improved Rankin's exponent $\delta_{1 / 2}^{+} \approx-0.065$ to $\varrho_{1 / 2}^{+} \approx-0.118$ (see (1.13) below for the definition of $\varrho_{r}^{+}$), as an application of his general result on the mean values of multiplicative functions and the fact that $F_{3}(s)$ and $F_{4}(s)$ are invertible for $\operatorname{Re} s \geq 1$, proven in the remarkable work of Kim \& Shahidi [11]. Although the result ([23, Corollary]) is stated only for Ramanujan's $\tau$-function, it is apparent that Tenenbaum's method applies to establish the upper bound for $S_{f}^{*}(x ; r)$ in $(1.11)$ below. It should be pointed out that Tenenbaum's approach is different from that of Rankin and does not give a lower bound for $S_{f}^{*}(x ; r)$.

The first aim of this paper is to improve the lower and upper bounds in (1.9), by generalizing Rankin's method to incorporate the aforementioned results of Kim \& Shahidi on $F_{3}(s)$ and $F_{4}(s)$.

Theorem 1. For any $f \in \mathrm{H}_{k}^{*}(N)$, we have

$$
x(\log x)^{\varrho_{r}^{\mp}} \ll S_{f}^{*}(x ; r) \ll x(\log x)^{\varrho_{r}^{ \pm}} \quad\left(r \in \mathscr{R}^{\mp}\right)
$$

for $x \geq x_{0}(f, r)$, where

$$
\mathscr{R}^{-}:=[0,1] \cup[2,3] \cup[4, \infty), \quad \mathscr{R}^{+}:=[1,2] \cup[3,4],
$$

and

$$
\left\{\begin{aligned}
\varrho_{r}^{-}:= & \frac{3^{r-1}-1}{2} \\
\varrho_{r}^{+}:= & \frac{102+7 \sqrt{21}}{210}\left(\frac{6-\sqrt{21}}{5}\right)^{r} \\
& +\frac{102-7 \sqrt{21}}{210}\left(\frac{6+\sqrt{21}}{5}\right)^{r}+\frac{4^{r}}{35}-1 .
\end{aligned}\right.
$$

The implied constants in (1.11) depend on $f$ and $r$.

The upper bound part in (1.11) is essentially due to Tenenbaum [23], since his method with a minor modification allows us to obtain this result. The lower bound part is new.

The following table illustrates progress on Rankin's (1.9) and the difference from the conjectured values (1.10).

\begin{tabular}{cccccccccc}
\hline$r$ & 0 & 0.5 & 1 & 1.5 & 2 & 2.5 & 3 & 3.5 & 4 \\
$\delta_{r}^{-}$ & -0.5 & -0.292 & 0 & 0.414 & 1 & 1.828 & 3 & 4.656 & 7 \\
$\varrho_{r}^{-}$ & -0.333 & -0.211 & 0 & 0.366 & 1 & 2.098 & 4 & 7.294 & 13 \\
$\theta_{r}$ & 0 & -0.151 & 0 & 0.358 & 1 & 2.104 & 4 & 7.278 & 13 \\
$\varrho_{r}^{+}$ & 0 & -0.118 & 0 & 0.350 & 1 & 2.111 & 4 & 7.257 & 13 \\
$\delta_{r}^{+}$ & 0 & -0.065 & 0 & 0.289 & 1 & 2.526 & 5.666 & 12.017 & 24.777 \\
\hline
\end{tabular}


In order to detect sign changes or cancellations among $\lambda_{f}(n)$, it is natural to study the summatory function

$$
S_{f}(x):=\sum_{n \leq x} \lambda_{f}(n)
$$

and compare it with (1.11). Investigation of the upper estimate for $S_{f}(x)$ has a long history. In 1927, Hecke [8] showed

$$
S_{f}(x) \ll_{f} x^{1 / 2}
$$

for all $f \in \mathrm{H}_{k}^{*}(N)$ and $x \geq 1$. Subsequent improvements came with the use of the identity

$$
\frac{1}{\Gamma(r+1)} \sum_{n \leq x}(x-n)^{r} a_{f}(n)=\frac{1}{(2 \pi)^{3}} \sum_{n \geq 1}\left(\frac{x}{n}\right)^{(k+3) / 2} a_{f}(n) J_{k+3}(4 \pi \sqrt{n x}),
$$

where $a_{f}(n):=\lambda_{f}(n) n^{(k-1) / 2}$ and $J_{k}(t)$ is the first kind Bessel function. Such an identity was first given by Wilton [26] for Ramanujan's $\tau$-function, and later generalized by Walfisz [24] to other forms. Let $\vartheta$ be a constant satisfying

$$
\left|\lambda_{f}(n)\right| \ll n^{\vartheta} \quad(n \geq 1) .
$$

Walfisz proved that

$$
S_{f}(x) \ll_{f} x^{(1+\vartheta) / 3} \quad(x \geq 1) .
$$

Inserting into (1.15) the values of $\vartheta$ from the historical record yields

$$
S_{f}(x) \ll_{f, \varepsilon} \begin{cases}x^{11 / 24+\varepsilon} & (\text { Kloosterman [12]), } \\ x^{4 / 9+\varepsilon} & \text { (Davenport [2], Salié [19]), } \\ x^{5 / 12+\varepsilon} & \text { (Weil [25]), } \\ x^{1 / 3+\varepsilon} & \text { (Deligne [4]), }\end{cases}
$$

for any $\varepsilon>0$. Hafner \& Ivić [6, Theorem 1] removed the factor $x^{\varepsilon}$ of Deligne's result. On the other hand, by combining Walfisz' method with his idea in the study of (1.7), Rankin [18] showed that

$$
S_{f}(x) \ll_{f, \varepsilon} x^{1 / 3}(\log x)^{\delta_{1 / 2}^{+}+\varepsilon}
$$

for any $\varepsilon>0$ and $x \geq 2$.

Here we propose a better bound, by combining Walfisz' method [24] and Tenenbaum's approach [23]. It is worth pointing out that Tenenbaum's method is not only to improve $\delta_{1 / 2}^{+}$to $\varrho_{1 / 2}^{+}$but also remove the $\varepsilon$ in (1.16).

Theorem 2. For $f \in \mathrm{H}_{k}^{*}(N)$, we have

$$
S_{f}(x) \ll x^{1 / 3}(\log x)^{\varrho_{1 / 2}^{+}}
$$

for $x \geq 2$, where the implied constant depends on $f$. 
In the opposite direction, Hafner \& Ivić [6, Theorem 2] proved that there is a positive constant $D$ such that

$$
S_{f}(x)=\Omega_{ \pm}\left(x^{1 / 4} \exp \left\{\frac{D\left(\log _{2} x\right)^{1 / 4}}{\left(\log _{3} x\right)^{3 / 4}}\right\}\right)
$$

where $\log _{r}$ denotes the $r$-fold iterated logarithm.

As an application of Theorems 1 and 2, we consider the quantities

$$
\mathscr{N}_{f}^{ \pm}(x):=\sum_{\substack{n \leq x \\ \lambda_{f}(n) \gtrless 0}} 1 .
$$

Very recently Kohnen, Lau \& Shparlinski [13, Theorem 1] proved

$$
\mathscr{N}_{f}^{ \pm}(x) \gg_{f} \frac{x}{(\log x)^{17}}
$$

for $x \geq x_{0}(f)\left({ }^{1}\right)$.

Here we propose a better bound.

Corollary 1. For any $f \in \mathrm{H}_{k}^{*}(N)$, we have

$$
\mathscr{N}_{f}^{ \pm}(x) \gg \frac{x}{(\log x)^{1-1 / \sqrt{3}}}
$$

for $x \geq x_{0}(f)$, where the implied constant depends on $f$. If we assume SatoTate's conjecture, then the exponent $1-1 / \sqrt{3} \approx 0.422$ can be improved to $2-16 /(3 \pi) \approx 0.302$.

In a joint paper with Lau [14], we shall remove the logarithmic factor by a completely different method.

2. Method of Rankin. Let $k \geq 2$ be an even integer, $N \geq 1$ be squarefree, $f \in \mathrm{H}_{k}^{*}(N)$ and $r>0$. Following Rankin's idea [17], we shall find two optimal multiplicative functions $\lambda_{f, r}^{ \pm}(n)$ such that

$$
\lambda_{f, r}^{\mp}\left(p^{\nu}\right) \leq\left|\lambda_{f}\left(p^{\nu}\right)\right|^{2 r} \leq \lambda_{f, r}^{ \pm}\left(p^{\nu}\right) \quad\left(r \in \mathscr{R}^{\mp}\right)
$$

for all primes $p$ and integers $\nu \geq 1$; furthermore, their associated Dirichlet series $\Lambda_{f, r}^{ \pm}(s)$ (see (2.8) below) in the half-plane Re $s \geq 1$ will be controlled by $F_{j}(s)$ for $j=1, \ldots, 4$. Then we can apply Tauberian theorems to obtain the asymptotic behaviour of the summatory functions of $\lambda_{f, r}^{ \pm}(n)$.

2.1. Construction of $\lambda_{f, r}^{ \pm}(n)$. For $\boldsymbol{a}:=\left(a_{1}, \ldots, a_{4}\right) \in \mathbb{R}^{4}$ and $r>0$, consider the function

$$
h_{r}(t ; \boldsymbol{a}):=t^{r}-a_{1} t-a_{2} t^{2}-a_{3} t^{3}-a_{4} t^{4} \quad(0 \leq t \leq 1)
$$

$\left({ }^{1}\right)$ It is worth indicating that they gave explicit values for the implied constant in $\ll$ and for $x_{0}(f)$. 
and let

$$
\kappa_{-}:=\frac{1}{4}, \quad \eta_{-}:=\frac{3}{4}, \quad \kappa_{+}:=\frac{6-\sqrt{21}}{20}, \quad \eta_{+}:=\frac{6+\sqrt{21}}{20} .
$$

In Subsection 2.3, we shall explain the reason behind this choice.

LEMMA 2.1. If the function $h_{r}(t ; \boldsymbol{a})$ defined by $(2.2)$ satisfies

$$
h_{r}^{\prime}\left(\kappa_{-} ; \boldsymbol{a}\right)=h_{r}^{\prime}\left(\eta_{-} ; \boldsymbol{a}\right)=h_{r}\left(\kappa_{-} ; \boldsymbol{a}\right)=h_{r}\left(\eta_{-} ; \boldsymbol{a}\right)=0,
$$

then

$$
a_{j}=a_{j}^{-}:=\frac{P_{j}^{-}\left(\kappa_{-}, \eta_{-}\right)-P_{j}^{-}\left(\eta_{-}, \kappa_{-}\right)}{\left(\kappa_{-}-\eta_{-}\right)^{3}}
$$

for $1 \leq j \leq 4$, where

$$
\begin{aligned}
& P_{1}^{-}(\kappa, \eta):=\{(4-r) \kappa+(r-2) \eta\} \kappa^{r-1} \eta^{2}, \\
& P_{2}^{-}(\kappa, \eta):=\left\{(2 r-8) \kappa^{2}+(1-r) \kappa \eta+(1-r) \eta^{2}\right\} \kappa^{r-2} \eta, \\
& P_{3}^{-}(\kappa, \eta):=\left\{(4-r) \kappa^{2}+(4-r) \kappa \eta+2(r-1) \eta^{2}\right\} \kappa^{r-2}, \\
& P_{4}^{-}(\kappa, \eta):=\{(r-3) \kappa+(1-r) \eta\} \kappa^{r-2} .
\end{aligned}
$$

Proof. This can be done by routine calculation.

LEMMA 2.2. If the function $h_{r}(t ; \boldsymbol{a})$ defined by $(2.2)$ is such that

$$
\left\{\begin{array}{l}
h_{r}^{\prime}\left(\kappa_{+} ; \boldsymbol{a}\right)=h_{r}^{\prime}\left(\eta_{+} ; \boldsymbol{a}\right)=0, \\
h_{r}\left(\kappa_{+} ; \boldsymbol{a}\right)=h_{r}\left(\eta_{+} ; \boldsymbol{a}\right)=h_{r}(1 ; \boldsymbol{a}),
\end{array}\right.
$$

then

$$
a_{j}=a_{j}^{+}:=\frac{P_{j}^{+}\left(\kappa_{+}, \eta_{+}\right)-P_{j}^{+}\left(\eta_{+}, \kappa_{+}\right)}{\left(\kappa_{+}-1\right)^{2}\left(\eta_{+}-1\right)^{2}\left(\kappa_{+}-\eta_{+}\right)^{3}}
$$

for $1 \leq j \leq 4$, where

$$
\begin{aligned}
P_{1}^{+}(\kappa, \eta):= & r \kappa^{r-1} \eta(\kappa-1)(\eta-\kappa)(\kappa \eta+2 \kappa+\eta)(\eta-1)^{2} \\
& +2\left(\kappa^{r}-1\right) \kappa \eta(\eta-1)^{2}\left(2 \kappa \eta+4 \kappa-\eta^{2}-2 \eta-3\right), \\
P_{2}^{+}(\kappa, \eta):= & r \kappa^{r-1}(\kappa-1)(\kappa-\eta)(\eta-1)^{2}\left(2 \kappa \eta+\kappa+\eta^{2}+2 \eta\right) \\
+ & \left(\eta^{r}-1\right)(\kappa-1)^{2}\left(8 \kappa \eta^{2}+4 \eta^{2}-\eta \kappa^{2}-2 \kappa \eta-3 \eta-\kappa^{3}-2 \kappa^{2}-3 \kappa\right), \\
P_{3}^{+}(\kappa, \eta):= & r \kappa^{r-1}(\kappa-1)(\kappa+2 \eta+1)(\eta-\kappa)(\eta-1)^{2} \\
& +2\left(\kappa^{r}-1\right)\left(2 \kappa^{2}+2 \kappa \eta-\eta^{2}-2 \eta-1\right)(\eta-1)^{2}, \\
P_{4}^{+}(\kappa, \eta):= & r \kappa^{r-1}(\kappa-1)(\kappa-\eta)(\eta-1)^{2}+\left(\eta^{r}-1\right)(\kappa-1)^{2}(3 \eta-\kappa-2) .
\end{aligned}
$$

Proof. This is done by routine calculation as well.

LEMMA 2.3. Let $\boldsymbol{a}^{ \pm}:=\left(a_{1}^{ \pm}, \ldots, a_{4}^{ \pm}\right)$, where the values of $a_{i}^{ \pm}$are given in Lemmas 2.1 and 2.2. Then for $0 \leq t \leq 1$ we have

$$
h_{r}\left(t ; \boldsymbol{a}^{-}\right) \gtrless 0 \quad \text { and } \quad h_{r}\left(t ; \boldsymbol{a}^{+}\right) \lessgtr h_{r}\left(1 ; \boldsymbol{a}^{+}\right) \quad \text { for } \quad r \in \mathscr{R}^{\mp} .
$$


Proof. We have

$$
h_{r}^{(4)}\left(t ; \boldsymbol{a}^{-}\right)=r(r-1)(r-2)(r-3) t^{r-4}-24 a_{4}^{-},
$$

so $h_{r}^{(4)}\left(t ; \boldsymbol{a}^{-}\right)$has at most one zero for $t>0$ and $h_{r}^{(i)}\left(t ; \boldsymbol{a}^{-}\right)$has at most $5-i$ zeros for $t>0(i=3,2,1,0)$. Since $h_{r}\left(\kappa_{-} ; \boldsymbol{a}^{-}\right)=h_{r}\left(\eta_{-} ; \boldsymbol{a}^{-}\right)=h_{r}\left(0 ; \boldsymbol{a}^{-}\right)$, it follows that $h_{r}^{\prime}\left(\xi_{-} ; \boldsymbol{a}^{-}\right)=h_{r}^{\prime}\left(\xi_{-}^{\prime} ; \boldsymbol{a}^{-}\right)=0$ for some $\xi_{-} \in\left(0, \kappa_{-}\right)$and $\xi_{-}^{\prime} \in\left(\kappa_{-}, \eta_{-}\right)$. Therefore $\xi_{-}, \kappa_{-}, \xi_{-}^{\prime}$ and $\eta_{-}$are the only zeros of $h_{r}^{\prime}\left(t ; \boldsymbol{a}^{-}\right)$ in $(0,1)$.

Now

$$
\begin{aligned}
& h_{r}^{\prime \prime}\left(\kappa_{-} ; \boldsymbol{a}^{-}\right)=8 \cdot 4^{-r}\left(2 r^{2}-2 r+3+2 r 3^{r-2}-11 \cdot 3^{r-2}\right), \\
& h_{r}^{\prime \prime}\left(\eta_{-} ; \boldsymbol{a}^{-}\right)=8 \cdot 4^{-r}\left(2 r^{2}-6 r-3-2 r 3^{r}+43 \cdot 3^{r-2}\right) .
\end{aligned}
$$

From these, it is easy to verify that

$$
h_{r}^{\prime \prime}\left(\kappa_{-} ; \boldsymbol{a}^{-}\right), h_{r}^{\prime \prime}\left(\eta_{-} ; \boldsymbol{a}^{-}\right) \begin{cases}\gtrless 0 & \text { if } r \in \mathscr{\mathscr { R }}^{\mp}, \\ =0 & \text { if } r=1,2,3,4,\end{cases}
$$

where $\stackrel{\circ}{\mathscr{R}}^{\mp}$ denotes the interior of $\mathscr{R}^{\mp}$. Hence $h_{r}\left(t ; \boldsymbol{a}^{-}\right)$takes its minimum (maximum, respectively) values in $[0,1]$ at $0, \kappa_{-}, \eta_{-}$when $r \in \stackrel{\circ}{\mathscr{R}}^{-}\left(r \in \stackrel{\circ}{\mathscr{R}}^{+}\right.$, respectively). Moreover, $h_{r}\left(t ; \boldsymbol{a}^{-}\right)$has local maxima (minima, respectively) at $\xi_{-}, \xi_{-}^{\prime}$ when $r \in \mathscr{\mathscr { R }}^{-}\left(r \in \stackrel{\circ}{\mathscr{R}}^{+}\right.$, respectively). This proves the assertion about $h_{r}\left(t ; \boldsymbol{a}^{-}\right)$.

Similarly we can prove the corresponding result on $h_{r}\left(t ; \boldsymbol{a}^{+}\right)$.

Now we define the multiplicative function $\lambda_{f, r}^{ \pm}(n)$ by

$$
\lambda_{f, r}^{\mp}\left(p^{\nu}\right):= \begin{cases}\sum_{0 \leq j \leq 4} 2^{2(r-j)} a_{j}^{\mp} \lambda_{f}(p)^{2 j} & \text { if } \nu=1 \text { and } r>0, \\ 0 & \text { if } \nu \geq 2 \text { and } r \in \mathscr{R}^{\mp}, \\ \left|\lambda_{f}\left(p^{\nu}\right)\right|^{2 r} & \text { if } \nu \geq 2 \text { and } r \in \mathscr{R}^{ \pm},\end{cases}
$$

where

$$
a_{0}^{-}:=0 \quad \text { and } \quad a_{0}^{+}:=1-a_{1}^{+}-a_{2}^{+}-a_{3}^{+}-a_{4}^{+} .
$$

In view of (1.6), we can apply Lemma 2.3 with $t=\left|\cos \theta_{f}(p)\right|$ to deduce that the inequality (2.1) holds for all primes $p$ and integers $\nu \geq 1$. By multiplicativity, this inequality also holds for all integers $n \geq 1$ (in place of $p^{\nu}$ ).

2.2. Dirichlet series associated to $\lambda_{f, r}^{ \pm}(n)$. For $f \in \mathrm{H}_{k}^{*}(N), r>0$ and $\operatorname{Re} s>1$, we define

$$
\Lambda_{f, r}^{ \pm}(s):=\sum_{n \geq 1} \lambda_{f, r}^{ \pm}(n) n^{-s}
$$


Next we shall study their analytic properties in the half-plane $\operatorname{Re} s \geq 1$ by using the higher order symmetric power $L$-functions $L\left(s, \operatorname{sym}^{m} f\right)$ associated to $f \in \mathrm{H}_{k}^{*}(N)$, due to Gelbart \& Jacquet [5] for $m=2$, and Kim \& Shahidi ([10], [11]) for $m=3,4,5,6,7,8$. Here the symmetric $m$ th power associated to $f$ is defined as

$$
L\left(s, \operatorname{sym}^{m} f\right):=\prod_{p} \prod_{0 \leq j \leq m}\left(1-\alpha_{f}(p)^{m-j} \beta_{f}(p)^{j} p^{-s}\right)^{-1}
$$

for $\operatorname{Re} s>1$, where $\alpha_{f}(p)$ and $\beta_{f}(p)$ are given by (1.3) and (1.4). According to the references mentioned above, the function $L\left(s, \operatorname{sym}^{m} f\right)$ for $m=2,3, \ldots, 8$ is invertible for $\operatorname{Re} s \geq 1$.

We start by studying $F_{1}(s), F_{2}(s), F_{3}(s)$ and $F_{4}(s)$, where $F_{r}(s)$ is defined by $(1.8)$.

Lemma 2.4. Let $k \geq 2$ be an even integer, $N \geq 1$ be squarefree and $f \in \mathrm{H}_{k}^{*}(N)$. For $j=1,2,3,4$ and $\operatorname{Re} s>1$, we have

$$
F_{j}(s)=\zeta(s)^{m_{j}} G_{j}(s) H_{j}(s),
$$

where

$$
m_{1}:=1, \quad m_{2}:=2, \quad m_{3}:=5, \quad m_{4}:=14,
$$

and

$$
\begin{aligned}
& G_{1}(s):=L\left(s, \operatorname{sym}^{2} f\right) \\
& G_{2}(s):=L\left(s, \operatorname{sym}^{2} f\right)^{3} L\left(s, \operatorname{sym}^{4} f\right) \\
& G_{3}(s):=L\left(s, \operatorname{sym}^{2} f\right)^{9} L\left(s, \operatorname{sym}^{4} f\right)^{5} L\left(s, \operatorname{sym}^{6} f\right) \\
& G_{4}(s):=L\left(s, \operatorname{sym}^{2} f\right)^{34} L\left(s, \operatorname{sym}^{4} f\right)^{20} L\left(s, \operatorname{sym}^{6} f\right)^{7} L\left(s, \operatorname{sym}^{8} f\right)
\end{aligned}
$$

are invertible for $\operatorname{Re} s \geq 1$. Here the function $H_{j}(s)$ admits a Dirichlet series convergent absolutely in $\operatorname{Re} s>1 / 2$ and $H_{j}(s) \neq 0$ for $\operatorname{Re} s=1$.

Proof. Write $x$ for the trace of a local factor of $L(s, f)$ (i.e. $\alpha_{f}(p)+\beta_{f}(p)$ ), and denote by $T_{n}(x)$ the polynomial which is the trace of its $n$th symmetric power. Then

$$
\begin{aligned}
& T_{2}=x^{2}-1, \\
& T_{4}=x^{4}-3 x^{2}+1, \\
& T_{6}=x^{6}-5 x^{4}+6 x^{2}-1, \\
& T_{8}=x^{8}-7 x^{6}+15 x^{4}-10 x^{2}+1,
\end{aligned}
$$


from which we deduce

$$
\begin{aligned}
& x^{2}=1+T_{2}, \\
& x^{4}=2+3 T_{2}+T_{4}, \\
& x^{6}=5+9 T_{2}+5 T_{4}+T_{6}, \\
& x^{8}=14+34 T_{2}+20 T_{4}+7 T_{6}+T_{8} .
\end{aligned}
$$

This implies (2.9). By the results on $L\left(s, \operatorname{sym}^{m} f\right)$ mentioned above, $G_{j}(s)$ is invertible for $\operatorname{Re} s \geq 1$.

Lemma 2.5. Let $k \geq 2$ be an even integer, $N \geq 1$ be squarefree and $f \in \mathrm{H}_{k}^{*}(N)$. For $r>0$ and $\operatorname{Re} s>1$, we have

$$
\Lambda_{f, r}^{ \pm}(s)=\zeta(s)^{\varrho_{r}^{ \pm}+1} H_{f, r}^{ \pm}(s),
$$

where

$$
\varrho_{r}^{ \pm}:=2^{2 r-8}\left(2^{8} a_{0}^{ \pm}+2^{6} a_{1}^{ \pm}+2^{4} \cdot 2 a_{2}^{ \pm}+2^{2} \cdot 5 a_{3}^{ \pm}+14 a_{4}^{ \pm}\right)-1
$$

and $H_{f, r}^{ \pm}(s)$ is invertible for $\operatorname{Re} s \geq 1$.

Proof. By definition (2.6), for $\operatorname{Re} s>1$ we can write

$$
\Lambda_{f, r}^{-}(s)=\prod_{p}\left(1+\sum_{0 \leq j \leq 4} 2^{2(r-j)} a_{j}^{-} \lambda_{f}(p)^{2 j} p^{-s}\right)=\prod_{0 \leq j \leq 4} F_{j}(s)^{2^{2(r-j)} a_{j}^{-}} H_{r}^{-}(s)
$$

for $r \in \mathscr{R}^{-}$, and

$$
\begin{aligned}
\Lambda_{f, r}^{-}(s) & =\prod_{p}\left(1+\sum_{0 \leq j \leq 4} 2^{2(r-j)} a_{j}^{-} \lambda_{f}(p)^{2 j} p^{-s}+\sum_{\nu \geq 2}\left|\lambda_{f}\left(p^{\nu}\right)\right|^{2 r} p^{-\nu s}\right) \\
& =\prod_{0 \leq j \leq 4} F_{j}(s)^{2^{2(r-j)} a_{j}^{-}} H_{r}^{-}(s)
\end{aligned}
$$

for $r \in \mathscr{R}^{+}$, where $F_{0}(s)=\zeta(s)$ is the Riemann zeta-function and $H_{r}^{-}(s)$ is a Dirichlet series absolutely convergent for $\operatorname{Re} s>1 / 2$ such that $H_{r}^{-}(s) \neq 0$ for $\operatorname{Re} s=1$. Now the desired result with the "-" sign follows from Lemma 2.4. The other part can be treated in the same way.

2.3. Optimization of $\lambda_{f, r}^{ \pm}(p)$ and choice of $\kappa_{ \pm}, \eta_{ \pm}$. If we regard $\kappa_{ \pm}, \eta_{ \pm}$ as parameters, the $\varrho_{r}^{ \pm}$given by (2.12) are functions of those parameters. We choose $\left(\kappa_{ \pm}, \eta_{ \pm}\right)$in $(0,1)^{2}$ optimally (they must be solutions of $\partial \varrho_{r}^{ \pm} / \partial \kappa=0$ and $\left.\partial \varrho_{r}^{ \pm} / \partial \eta=0\right)$, which can be done by using formal calculation via Maple. Their values are given by (2.3).

3. Proof of Theorem 1. In view of Lemma 2.5 and a classical fact on $\zeta(s)$, we can write

$$
\Lambda_{f, r}^{ \pm}(s)=\frac{H_{f, r}^{ \pm}(1)}{(s-1) \varrho_{r}^{ \pm}+1}+g_{f, r}^{ \pm}(s)
$$


in some neighbourhood of $s=1$ with $\operatorname{Re} s>1$, where $H_{f, r}^{ \pm}(1) \neq 1$ and $g_{f, r}^{ \pm}(s)$ is holomorphic at $s=1$. Since $\lambda_{f, r}^{ \pm}(n) \geq 0$, we can apply Delange's tauberian theorem [3] to write

$$
\sum_{n \leq x} \lambda_{f, r}^{ \pm}(n) \sim H_{f, r}^{ \pm}(1) x(\log x)^{\varrho_{r}^{ \pm}} \quad(x \rightarrow \infty) .
$$

Now Theorem 1 follows from (2.1) and (3.2).

4. Proof of Theorem 2. By (3.1), it follows that

$$
\prod_{p}\left(1+\sum_{\nu \geq 1} \frac{\lambda_{f, r}^{ \pm}\left(p^{\nu}\right)}{p^{\nu \sigma}}\right)=\frac{H_{f, r}^{ \pm}(1)}{(\sigma-1)^{ \pm}+1}+g_{f, r}^{ \pm}(\sigma)
$$

for $\sigma>1$. From this, (2.6), (2.7) and Deligne's inequality, we deduce that

$$
\sum_{p} \frac{\lambda_{f, r}^{ \pm}(p)}{p^{\sigma}}=\left(\varrho_{r}^{ \pm}+1\right) \log (\sigma-1)^{-1}+C_{f, r}^{ \pm}+o(1) \quad(\sigma \rightarrow 1+),
$$

where $C_{f, r}^{ \pm}$is some constant.

On the other hand, the prime number theorem implies, by partial integration, that

$$
\sum_{p} p^{-\sigma}=\log (\sigma-1)^{-1}+C+o(1) \quad(\sigma \rightarrow 1+)
$$

where $C$ is an absolute constant. Thus the preceding relation can be written as

$$
\sum_{p} \frac{\lambda_{f, r}^{ \pm}(p)-\left(\varrho_{r}^{ \pm}+1\right)}{p^{\sigma}}=C_{f, r}^{ \pm}+\left(\varrho_{r}^{ \pm}+1\right) C+o(1) \quad(\sigma \rightarrow 1+) .
$$

According to Exercise II.7.8 of [22], the formula (4.1) implies

$$
\sum_{p} \frac{\lambda_{f, r}^{ \pm}(p)-\left(\varrho_{r}^{ \pm}+1\right)}{p}=C_{f, r}^{ \pm}+\left(\varrho_{r}^{ \pm}+1\right) C .
$$

Hence

$$
\sum_{p \leq x} \frac{\lambda_{f, r}^{ \pm}(p)}{p}=\left(\varrho_{r}^{ \pm}+1\right) \log _{2} x+C_{f, r}^{ \pm}+\left(\varrho_{r}^{ \pm}+1\right) C+o(1) \quad(x \rightarrow \infty) .
$$


Now we apply a well known result of Shiu [20] and (2.1) to write

$$
\begin{aligned}
\sum_{x \leq n \leq x+z}\left|\lambda_{f}(n)\right|^{2 r} & \ll \frac{z}{\log x} \exp \left(\sum_{p \leq x} \frac{\left|\lambda_{f}(p)\right|^{2 r}}{p}\right) \\
& \ll \frac{z}{\log x} \exp \left(\sum_{p \leq x} \frac{\lambda_{f, r}^{+}(p)}{p}\right) \\
& \ll z(\log x)^{\varrho_{r}^{+}}
\end{aligned}
$$

for $r \in \mathscr{R}^{-}$, any $\varepsilon>0, x \geq x_{0}(\varepsilon)$ and $x^{1 / 4} \leq z \leq x$. We use this with $r=1 / 2$ in (9) of [18]; then the first term on the right-hand side of (10) of [18] is replaced by $x^{1 / 2} z^{-1 / 2}(\log x)^{\varrho_{1 / 2}^{+}}$. Applying (4.2) with $r=1 / 2$ again to the second term on the right-hand side of (10) of [18] yields

$$
S_{f}(x) \ll x^{1 / 2} z^{-1 / 2}(\log x)^{\varrho_{1 / 2}^{+}}+z(\log x)^{\varrho_{1 / 2}^{+}} .
$$

Taking $z=x^{1 / 3}$, we obtain the required result when the level is $N=1$. The general case can be treated in much the same way as indicated in [18].

5. Proof of Corollary 1. By comparing (1.17) and the lower bound part in (1.11) with $r=1 / 2$, it is easy to deduce that

$$
\sum_{\substack{n \leq x \\ \lambda_{f}(n) \gtrless 0}}\left|\lambda_{f}(n)\right| \gg_{f} x(\log x)^{\varrho_{1 / 2}^{-}}
$$

for $x \geq x_{0}(f)$. Since $\varrho_{1 / 2}^{-}=-(1-1 / \sqrt{3}) / 2$ and $\varrho_{1}^{+}=0$, a simple application of the Cauchy-Schwarz inequality yields the desired estimate.

The second assertion can be obtained by noticing that $\theta_{1 / 2}=8 /(3 \pi)-1$.

Acknowledgments. The author would like to thank Winfried Kohnen for sending the paper [13] and Yuk Kam Lau for his many suggestions that improved the writing of this paper.

\section{References}

[1] L. Clozel, M. Harris and R. Taylor, Automorphy for some $\ell$-adic lifts of automorphic mod $\ell$ Galois representations, preprint.

[2] H. Davenport, On certain exponential sums, J. Reine Angew. Math. 169 (1932), $158-176$.

[3] H. Delange, Généralisation du théorème de Ikehara, Ann. Sci. École Norm. Sup. 71 (1954), 213-242.

[4] P. Deligne, La conjecture de Weil, I, II, Publ. Math. IHES 48 (1974), 273-308, 52 (1981), 313-428.

[5] S. Gelbart and H. Jacquet, A relation between automorphic representations of GL(2) and GL(3), Ann. Sci. École Norm. Sup. (4) 11 (1978), 471-542. 
[6] J. L. Hafner and A. Ivić, On sums of Fourier coefficients of cusp forms, Enseign. Math. (2) 35 (1989), 375-382.

[7] M. Harris, N. Shepherd-Barron and R. Taylor, A family of Calabi-Yau varieties and potential automorphy, preprint.

[8] E. Hecke, Theorie der Eisensteinsche Reihen höherer Stufe und ihre Anwendung auf Funktionentheorie und Arithmetik, Abh. Math. Sem. Univ. Hamburg 5 (1927), 199-224.

[9] H. Iwaniec, Topics in Classical Automorphic Forms, Grad. Stud. Math. 17, Amer. Math. Soc., Providence, RI, 1997.

[10] H. H. Kim and F. Shahidi, Functorial products for $\mathrm{GL}_{2} \times \mathrm{GL}_{3}$ and the symmetric cube for $\mathrm{GL}_{2}$ (with an appendix by C. J. Bushnell and G. Henniart), Ann. of Math. (2) 155 (2002), 837-893.

[11] - - -, Cuspidality of symmetric powers with applications, Duke Math. J. 112 (2002), 177-197.

[12] H. D. Kloosterman, Asymptotische Formeln für die Fourierkoeffizienten ganzer Modulformen, Abh. Math. Sem. Univ. Hamburg 5 (1927), 337-352.

[13] W. Kohnen, Y.-K. Lau and I. E. Shparlinski, On the number of sign changes of Hecke eigenvalues of newforms, J. Austral. Math. Soc. 85 (2008), 87-94.

[14] Y.-K. Lau and J. Wu, The number of Hecke eigenvalues of same signs, Math. Z., to appear.

[15] C. J. Moreno and F. Shahidi, The fourth moment of Ramanujan $\tau$-function, Math. Ann. 266 (1983), 233-239.

[16] R. A. Rankin, Sums of powers of cusp form coefficients, ibid. 263 (1983), 227-236.

[17] —, Sums of powers of cusp form coefficients, II, ibid. 272 (1985), 593-600.

[18] - Sums of cusp form coefficients, in: Automorphic Forms and Analytic Number Theory (Montréal, PQ, 1989), Univ. Montréal, Montréal, QC, 1990, 115-121.

[19] H. Salié, Zur Abschätzung der Fourierkoeffizienten ganzer Modulformen, Math. Z. 36 (1933), 263-278.

[20] P. Shiu, A Brun-Titchmarsh theorem for multiplicative functions, J. Reine Angew. Math. 313 (1980), 161-170.

[21] R. Taylor, Automorphy for some $\ell$-adic lifts of automorphic mod $\ell$ Galois representations, II, preprint, 2006.

[22] G. Tenenbaum (en collaboration avec J. Wu), Exercices corrigés de théorie analytique et probabiliste des nombres, Cours Spécialisés 2, Soc. Math. France, 1996.

[23] G. Tenenbaum, Remarques sur les valeurs moyennes de fonctions multiplicatives, Enseign. Math. (2) 53 (2007), 155-178.

[24] A. Walfisz, Über die Koeffizientensummen einiger Modulformen, Math. Ann. 108 (1933), 75-90.

[25] A. Weil, On some exponential sums, Proc. Nat. Acad. Sci. U.S.A. 34 (1948), 204-207.

[26] J. R. Wilton, A note on Ramanujan's arithmetical function $\tau(n)$, Proc. Cambridge Philos. Soc. 25 (1928), 121-129.

School of Mathematical Sciences

Shandong Normal University

Jinan, Shandong 250100, China
Institut Élie Cartan Nancy (IECN), CNRS

Nancy-Université, INRIA

Boulevard des Aiguillettes, B.P. 239

54506 Vandœuvre-lès-Nancy, France

E-mail:wujie@iecn.u-nancy.fr 